\title{
Development of a Web System-based Geographic Information System Technologies to Mapping Electromagnetic Fields: First Developments
}

\section{Desarrollo de un Sistema Web basado en Tecnologías de Sistemas de Información Geográfica para Mapeo de Campos Electromagnéticos: Primeros Desarrollos}

\section{Pedro Infante ${ }^{1}$, Alex Quingatuña ${ }^{1}$, Santiago Nogales ${ }^{1}$, Blanca Hidalgo¹, Tony Flores², and Fidel Gilard ${ }^{3}$}

Corresponding Author:

Pedro Infante

pinfante@espoch.edu.ec

Received: 4 December 2018

Accepted: 5 December 2018

Published: 27 December 2018

Publishing services provided by Knowledge E

(c) Pedro Infante et al. This article is distributed under the terms of the Creative Commons Attribution License, which permits unrestricted use and redistribution provided that the original author and source are credited.

Selection and Peer-review under the responsibility of the SIIPRIN-CITEGC Conference Committee.

\section{S OPEN ACCESS}

${ }^{1}$ Facultad de Informática y Electrónica, Escuela Superior Politécnica de Chimborazo, ECo60154 Riobamba, Ecuador

${ }^{2}$ Unidad de Investigación, Instituto Superior Pedagógico Jaime Roldós Aguilera,EC060401 Colta, Ecuador

${ }^{3}$ Centro Nacional de Electromagnetismo, Universidad de Oriente,Santiago de Cuba, Cuba

\section{Abstract}

The purpose of this research work is to make decisions about the exposure levels of people to radiation, for which, the Web System based on GIS technology (DECOMAPS) was developed for mapping electromagnetic fields in the city of Riobamba. This system executes analysis, search, creation, automation of processes, and mapping activities of electromagnetic fields. The system was developed on an agile development methodology called SCRUM, which allows to create an optimal and interactive work environment between the product owner and the developers in order to create a quality system. Many technological tools were applied by the developer of this system. Once successfully completed, the system was subjected to tests of functionality and usability of the final product through quality metrics established by ISO 9126-3, where it was determined that the system is $93.64 \%$ functional, in addition to a $94.40 \%$ in usability. Concluding that the system is functional and can be implemented as a contribution in research to be developed in the future on the effects of electromagnetic fields on the human body.

\section{Resumen}

El presente trabajo de investigación tiene como propósito tomar decisiones sobre los niveles de exposición de la gente a la radiación, para lo cual, el Sistema Web basado en tecnología GIS (DECOMAPS) fue desarrollado para mapeo de campos electromagnéticos en la ciudad de Riobamba. Este sistema ejecuta análisis, búsqueda, creación, automatización de procesos y actividades de mapeo de campos 
electromagnéticos. El sistema fue desarrollado sobre una metodología de desarrollo ágil llamada SCRUM, el cual permite crear un ambiente de trabajo óptimo e interactivo entre el propietario del producto y los desarrolladores en orden de crear un sistema de calidad. Muchas herramientas tecnológicas fueron aplicadas por el desarrollador de este sistema. Una vez completado exitosamente, el sistema fue sometido a pruebas de funcionalidad y usabilidad del producto final a través de métricas de calidad establecidas por la norma ISO 9126-3, donde se determinó que el sistema es 93,64\% funcional, además de un $94,40 \%$ en usabilidad. Concluyendo que el sistema es funcional y se puede implementar como una contribución en las investigaciones a desarrollarse a futuro sobre las afectaciones de los campos electromagnéticos sobre el cuerpo humano.

Keywords: OpenGeo Suite, Java, SCRUM, ISO 9126-3, DECOMAPS

Palabras clave: OpenGeo Suite, Java, SCRUM, ISO 9126-3, DECOMAPS

\section{Introducción}

El aumento de antenas transmisoras de radio, televisión y estaciones base de telefonía celular instaladas en sectores urbanos han generado un beneficio en el área de las telecomunicaciones, pero dicho aumento trae como consecuencia, una mayor radiación electromagnética-definida como la propagación de campos electromagne区ticos (EMF) mediante ondas a partir de una fuente- [1], a la cual están expuestas las personas cercanas a estos sectores, existiendo una preocupación en la ciudadanía por posibles efectos adversos en la salud. Se conoce que, de acuerdo al Grupo Bioiniciativa [2], los EMF están relacionados con el desarrollo de cáncer, leucemia, tumores cerebrales, etc., que han afectado a aquellas personas que han vivido a cortas distancias de antenas o estaciones base de transmisión. No obstante, se han descrito otras enfermedades que parecen tener relación con esta; tales como: irritabilidad, depresión, pérdida de memoria y mareos, entre otras, por lo cual, se ha visto necesario el estudio del impacto de los EMF en el país.

Debido a los riesgos antes mencionados, varias organizaciones, entre ellas la International Commission on Non-lonizing Radiation Protection (ICNIRP) y International Telecommunication Union (ITU) han planteado normativas y directrices que establecen ciertos límites de radiación a la exposición ocupacional y poblacional a los que puede 
estar expuesto el ser humano [3], [4]. Pero en muchos de los casos, el cumplimiento de estas recomendaciones no es aplicada en su totalidad por las empresas de telefonía celular.

Estudios realizados en la ciudad de Cali determinaron que los niveles de inmisión de densidad de potencia, aunque en su mayoría estaban por debajo de los límites permitidos (96\%), existían lugares donde los niveles de EMF superaron los límites establecidos por las entidades regulatorias, las cuales realizan constantes estudios de medición, ayudados de herramientas, entre ellas dispositivos medidores de radiación, Sistemas de Posicionamiento Global (GPS), Sistemas de Información Geográfica (GIS) [5]. Este mismo tipo de estudios de mediciones y el mapeo electromagne区ticos de la radiación de las antenas se realizaron en la ciudad de Riobamba utilizando herramientas de software existentes en el mercado como Radio Mobile y Open Street Map [6], con la finalidad de determinar si los rangos de radiación generada están dentro de los límites de radiación EMF de acuerdo a normativas internacionales tales como ICNIRP.

Uno de los procedimientos empleados para el control y estudio de los niveles de radiación es el mapeo de EMF, el cual consiste en realizar mediciones y cálculos utilizando equipos y personal capacitado, además de disponer de los datos obtenidos para utilizarlos en la toma de decisiones. Estos datos deben ser lo más precisos posibles, debido a que estos influirán directamente en la salud de las personas que están expuestas a la radiación de EMF producidos por las antenas.

En Ecuador se han realizado estudios basados en mediciones de EMF [6], sin embargo, la no existencia de aplicaciones GIS que ayuden en la representación y procesamiento de estos datos obtenidos [7] ha puesto en evidencia la creación de un sistema informático que ayude en el análisis e interpretación de resultados de las mediciones, y con ello ayude en la toma de decisiones sobre el porcentaje recomendable al que deberi冈a estar expuesto el ser humano, para lo cual, se ha considerado el desarrollo de una aplicacio®n georeferencial mediante el uso de herramientas GIS con el objetivo de conseguir un producto de calidad que satisfaga las necesidades planteadas por el usuario [8].

La Seccio囚n I hace una introduccio®n sobre la problema冈tica, para en la Seccio囚n II justificarla mediante una bu囚squeda exhaustiva de informacio囚n sobre los diferentes sistemas de informacio®n geogra冈fica y sistemas Web. Luego, en la Secciođn III se describe la estructura y la metodologi冈a para la implementacio囚n del nuevo sistema. Despue冈s, en la Seccio囚n IV presentamos los resultados sobre el funcionamiento, y; proponemos un Sistema para el mapeo de EMF. Finalmente, en la Seccioßn $V$ concluimos este trabajo. 


\section{Marco Teórico}

\subsection{Sistemas de Información Geográfica (GIS)}

Las GIS son tecnologi冈as que permite gestionar y analizar informacioØn espacial. Puede definirse como un conjunto de software, hardware, metodologi冈as, datos y usuarios, perfectamente integrados, de forma que hace posible la recoleccio®n, almacenamiento, procesamiento y ana囚lisis de datos georeferenciados, asi $₫$ como la produc-

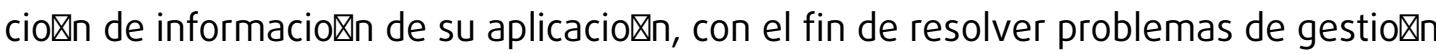
y de planificacio囚n [1]. Esto ha trai『do un nuevo punto de vista y un enorme beneficio para la investigacio囚n y desarrollo en el dominio del uso las GIS, que es el manejo de la radiacioßn electromagne区tica en las ciudades.

En comparacio囚n con los GIS tradicionales, las Web GIS tienen ventajas como la independencia de la plataforma, bajo costo de desarrollo, uso amigable, fa冈cil actualizacio囚n del sistema, equilibrio de carga, entre otras.

\subsubsection{Caracteri冈sticas y Prestaciones de los GIS}

Algunas de las caracteri冈sticas principales de los GIS son: a) Visualizacio囚n de infor-

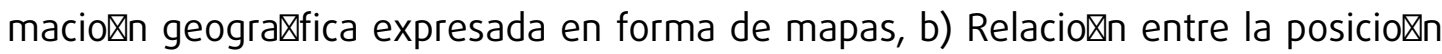
un elemento geogra冈fico, representado por puntos, li冈neas, poli冈gonos y su informacio®n tema冈tica asociada, c) Elevado nu囚mero de procedimientos de geometri冈a computacional, de algoritmos informa囚ticos, de me冈todos cuantitativos, etc., d) Almacena relaciones espaciales entre diferentes elementos [9].

Las prestaciones que los GIS suelen proporcionar son: a) Entrada y captura de datos, b) Administracio囚n y organizacio囚n informa区tica de los archivos de geodatos, c) Edi-

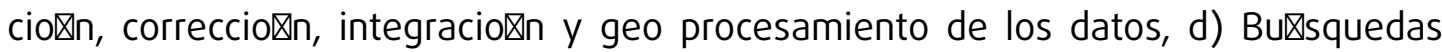
o selecciones, e) Ana区lisis estadi凶stico, f) Modelado y reconstruccioखn de aspectos realistas, g) Elaboracio囚n y visualizacio囚n de mapas bi y tridimensionales, h) Servicio remoto de informacioßn geogra囚fica bajo demanda de los usuarios, i) Impresio囚n y exportacio®n de mapas, gra冈ficos, datos y realidad virtual del territorio.

\subsection{Plataforma OpenGeo Suite}

OpenGeo Suite es una plataforma completa de aplicaciones geogra冈ficas basadas en un conjunto de herramientas de Java enfocadas a las tecnologi冈as GIS. Contiene lo 
necesario para la creaciođn de sistemas en la web, integra una base de datos capaz de gestionar datos espaciales georeferenciales, un servidor de aplicaciones y un cliente API [10]. La Figura 1 muestra la relacio®n entre algunos de los componentes que conforman la aplicacio囚n OpenGeo Suite (GeoServer, OpenLayer, PostGIS).

\subsubsection{Componente GeoServer}

Es un servidor web de co囚digo abierto desarrollado en Java, permite utilizar mapas y datos de diferentes formatos para sistemas web, ya sean clientes web ligeros, o programas GIS desktop. Usa Restlet como framework para los servicios REST que proporciona. Incluye Jetty como servidor embebido, e incluye GeoWebCache, un componente de cacheado basado en Java similar a TileCache [11].

\subsubsection{Componente OpenLayer}

Es una herramienta gratuita bajo licencia tipo BSD, permite visualizar un mapa dina冈mico en una pa囚gina web, OpenLayer es un proyecto de OpenSourceGeospatialFoundation, escrito en JavaScript. Implementa servicios web del consorcio OpenGIS de Mapping (WMS) y Web FeatureService (WFS). [12]

\subsubsection{Componente PostGIS}

Es una extensio囚n para el manejo de objetos geogra冈ficos (geodatabase) dentro de una base de datos PostgreSQL, el desarrollo de la aplicacio囚n se basa en esto, ya que posee mayor beneficio y menor costo, debido a que esta publicado bajo una licencia distribucio囚n libre GNU. PostGIS es estable, ra囚pido, compatible con esta冈ndares y actualmente es la base de datos espacial de co囚digo abierto ma冈s ampliamente utilizada. La administracioßn de la base de datos es posible a trave冈s de PgAdmin y phpPgAdmin, entre otros. Es posible importar y exportar datos mediante herramientas en li冈nea de comandos o a trave冈s de clientes SIG de escritorio o web. [13]

\subsubsection{Ventajas de OpenGeo Suite en el desarrollo de sistemas web georeferenciales}

Para la implementacio囚n del sistema DECOMAPS se selecciono® la plataforma en desarrollo de sistemas web georeferenciales OpenGeo Suite, por su flexibilidad al 


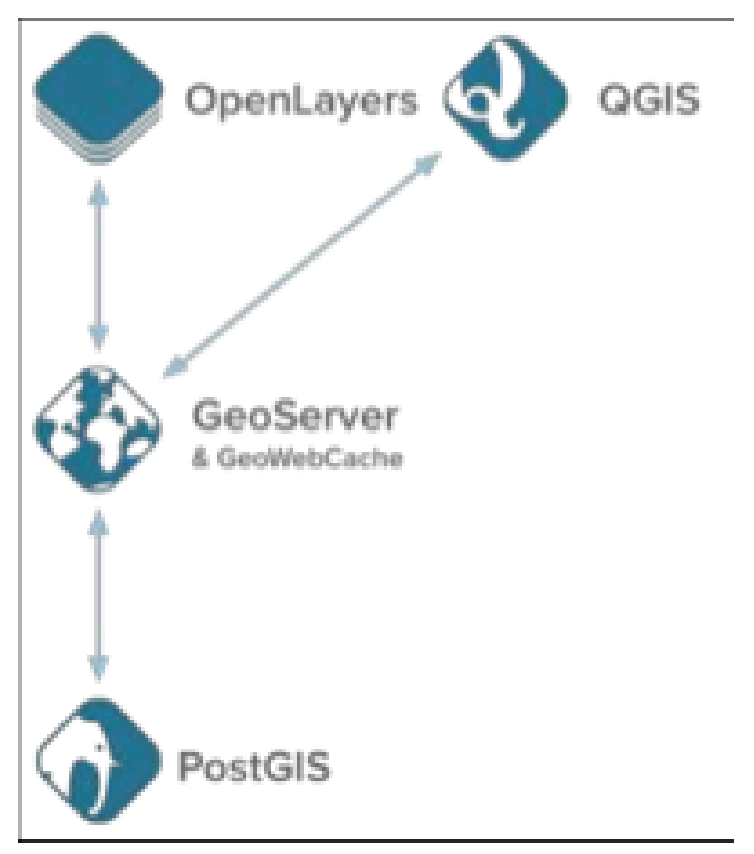

FIgura 1: Componentes de OpenGeo Suite.

unir componentes geogra冈ficos y sistemas de base de datos, salida de datos en varios formatos de servicios web, arquitectura web, escalabilidad, y soporte para varios sistemas operativos.

\subsection{Herramientas Informa冈ticas}

\subsubsection{Java}

Es un lenguaje de programacio囚n orientado a objetos, soportado por todos los sistemas operativos y dispositivos mo囚viles existentes. Fue desarrollado por Sun Microsystems y permite la creacio囚n de todo tipo de aplicaciones como de escritorio, web o mo囚viles [14].

\subsubsection{Apache Tomcat}

Es un servidor web basado en el lenguaje Java creada para ejecutar Java Servlet y JavaServer Pages (JSP), mediante un ana冈lisis comparativo de los servidores Web que soportan Java, en el cual se compararon las ventajas y desventajas de los servidores, se determino® que en su mayori冈a todos son eficientes, pero se decidio® por Apache Tomcat por motivos de costos y la facilidad para la configuracio囚n [15]. 


\subsubsection{Eclipse Mars IDE}

Es el IDE seleccionado ya que esta冈 compuesto por un conjunto de herramientas de programacio囚n de co囚digo abierto, usado en su mayori冈a para el desarrollo en aplicaciones de lenguaje Java, por su gran integracio囚n entre servidores y motores de base de datos. Adema囚s, Eclipse funciona en todos los sistemas operativos por lo que es muy beneficioso [16].

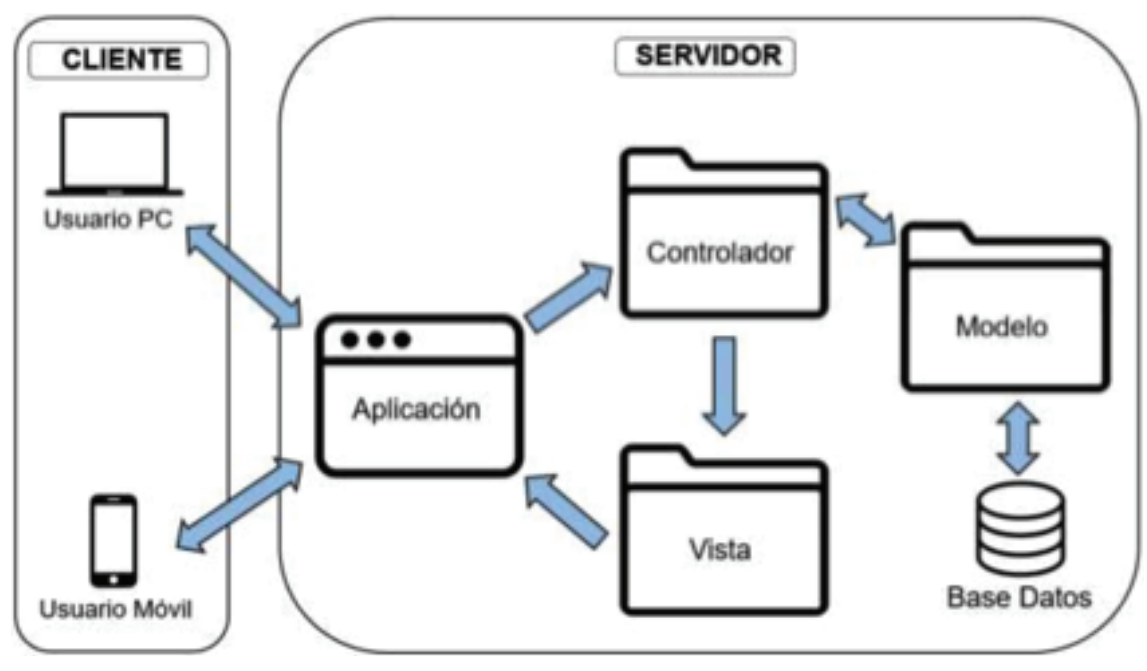

Figura 2: Arquitectura del sistema.

\subsection{Arquitectura del sistema}

El sistema fue construido aplicando la arquitectura Cliente - Servidor, y haciendo uso de los patrones Modelo Vista Controlador (MVC) [17], el mismo que ha sido seleccionado y disen囚ado con base en objetivos (requerimientos) y restricciones que se han presentado en el proyecto. Su fundamento es la separacio囚n del co囚digo en tres capas diferentes, acotadas por su responsabilidad, en lo que se llaman Modelos, Vistas y Controladores.

\subsubsection{Modelo}

Es la capa donde se trabaja con los datos, contiene todas las funciones que accedera冈n a las tablas y hara冈n las correspondientes inserciones, actualizaciones, eliminaciones, cargas, etc. 


\subsubsection{Vista}

Es la capa que contiene todos los formularios que son visualizados dentro de las interfaces de usuario, permitiendo con ello la interaccio囚n entre el usuario y la aplicacio囚n.

\subsubsection{Controlador}

Es la capa que sirve de enlace entre las vistas y los modelos, respondiendo a los mecanismos que puedan requerirse para implementar las necesidades de nuestra aplicacio囚n.

La Figura 2 muestra la colaboracio囚n entre los elementos que forman una aplicacio囚n MVC, haciendo uso de la arquitectura cliente - servidor. Como se puede ver, el controlador envi冈a y recepta las peticiones realizadas por el cliente, el modelo procesa las consultas SQL y la vista muestra el contenido en un formato legible para el cliente.

\subsection{Metodologi冈a empleada para la creación de la aplicación}

Mediante el ana冈lisis realizado a las caracteri冈sticas principales de cada una de las metodologi冈as a冈giles y tradicionales [18] que se utilizan para el desarrollo de proyectos de software, el proyecto ha sido implementado mediante el uso y aplicacio囚n de la metodologi冈a a囚gil Ilamada SCRUM, poniendo e囚nfasis en el trabajo en equipo y obteniendo mejores resultados de productividad.

\subsubsection{Metodologi区a de desarrollo SCRUM}

Es una metodologi冈a a冈gil, usada porque minimiza los riesgos durante la realizacio®n de un proyecto, pero de manera colaborativa. Entre las ventajas se encuentran la productividad, calidad y el seguimiento diario con el que se realiza los avances del proyecto, logrando que los integrantes este冈n unidos, comunicados y que el cliente vaya viendo los avances.

\subsubsection{Fases de SCRUM}

La tabla I muestra las actividades a realizarse en cada fase para el funcionamiento de la metodologi冈a SCRUM. 


\subsubsection{Participantes de SCRUM}

Son aquellos que forman parte en el desarrollo del proyecto y tienen sus caracteri囚sticas propias, estos son: Product Owner (Responsable del proyecto), SCRUM Master (Liøder de las reuniones), SCRUM Team (Desarrollador del proyecto) y Clientes (Receptor del producto final).

TABLA 1: Fases de SCRUM.

Fases
Product Backlog
Sprint Backlog
Sprint Planning Meeting
Daily SCRUM
Spring Review
Spring Retrospective

\section{Descripción}

Lista de requerimientos del Proyecto

Actividad del Proyecto

Reunión al inicio de cada Sprint

Reunión breve diaria durante el Sprint

Revisión del Sprint terminado

Retroalimentación del Sprint terminado

\subsection{ISO/IEC 9126-3 Calidad del software}

El esta冈ndar internacional ISO/IEC 9126-3 [19] define las caracteri冈sticas de calidad y el modelo del proceso de evaluacio囚n del software basado en dos puntos importantes: a) Los requisitos del software constituyen el fundamento para medir la calidad; $y, b$ ) Los esta冈ndares utilizados definen un conjunto de criterios que gui冈an la manera en que el software se somete a evaluaciođn. Para nuestro sistema DECOMAPS se han seleccionado dos caracteri凶sticas, las cuales se aplicara冈n al modelo de proceso para la evaluacioßn de calidad.

1. Funcionalidad.- Caracteri凶stica seleccionada por las condiciones especi凶ficas que debe cumplir el sistema.

2. Usabilidad.- Caracteri冈stica seleccionada con la finalidad de evaluar la interaccio®n del sistema con el usuario.

\section{Desarrollo de la Aplicación}

Con el desarrollo del sistema web para el mapeo de las radiaciones de EMF en la ciudad de Riobamba, se podra gestionar los datos de mediciones de los niveles mi囚nimo,

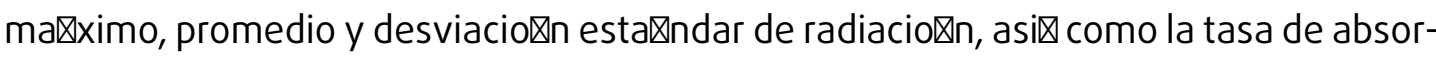
cio囚n especi凶fica (SAR) en determinadas partes del cuerpo humano y compararlos con 
los valores li冈mites permitidos por las normas internacionales, tales como las proporcionadas por la ICNIRP. El sistema estara区 compuesto de los siguientes mo囚dulos:

- Mo囚dulo de Creacio®n y Autenticacio囚n de Usuarios

- Moødulo de Mapas

- Mo囚dulo de Ingreso de Datos

- Mo囚dulo de Ubicacio囚n Geogra冈fica de Estaciones Base

- Mo囚dulo de Procesamiento de datos

- Mo囚dulo de Reportes

\subsection{SCRUM - Fase de Planificacio囚n}

\subsubsection{Identificacio囚n de Usuarios IIIly Roles}

El equipo involucrado en la gestioßn y desarrollo del proyecto esta囚 conformado por el director del proyecto (SCRUM Master), el propietario del producto (Product Owner), y el equipo de desarrollo.

\subsubsection{Tipos de Usuarios y Roles}

Los usuarios son todas aquellas personas involucradas directamente en el uso del sistema, por lo cual se definira冈n a continuacio囚n un tipo de usuario y su rol en el sistema.

\subsubsection{Actividades del proyecto}

Las actividades realizadas antes, durante y despue冈s del desarrollo del presente proyecto.

\subsubsection{Producto BackLog}

Las historias de usuario (Product Backlog), es el conjunto de requisitos generados por el equipo SCRUM para definir las actividades de cada miembro del equipo de desarrollo y determinar el nu囚mero de Sprints (iteraciones) del proyecto. 


\subsubsection{Sprint BackLog}

Las iteraciones del proyecto (Sprint) cuenta de un cierto nu囚mero de historias de usuario agrupadas para ser completadas en un rango de tiempo (cuatro semanas).

\subsubsection{Cronograma de actividades}

Para el cumplimiento de los Sprints se ha establecido un cronograma de actividades estimado con una duracioßn de 20 semanas, trabajando 8 horas diarias de lunes a viernes.

\subsubsection{Reuniones SCRUM}

La metodologi囚a utilizada indica que el equipo SCRUM puede mantener reuniones frecuentemente para definir actividades, requisitos y caracteri凶sticas de uखltimo momento que pueda requerir el Product Owner o propietario del producto.

\subsection{SCRUM - Fase de Desarrollo}

\subsubsection{Descripcio囚n de las Capas de la Aplicacio囚n}

El sistema contara区 con una estructura basada en 3 capas (MVC). Cada procesador cuenta con un servidor web Apache TomCat v.8.026 alojado en Ubuntu 15.01.

1. Modelo: donde se ejecutan todas sentencias SQL y los procesos de conexio囚n a la base de datos.

2. Controlador: donde se ejecutan las validaciones necesarias para mantener la integridad de los datos adema冈s garantiza la creacio囚n de Aplicaciones Empresariales con Calidad.

3. Vista: donde se aloja toda la interfaz de usuario y ciertas validaciones.

\subsubsection{Esta冈ndar de Codificacio $\ n$}

Para proporcionar una correcta utilizacioØn de los te冈rminos sema冈nticos, procesos de escritura de co囚digo, se seleccionó el esta囚ndar de codificacio囚n J2EE CHECKLIST. 


\subsubsection{Disen囚o de la Base de Datos}

Se realizo® un ana区lisis previo de la funcionalidad que tendra囚 el sistema, garantizando que los datos sera冈n manejados de mejor manera. Se empleo® el modelo relacional, y esta $₫$ conformada por un total de 8 tablas.

\subsubsection{Diccionario de Datos}

Es una representacio®n de las caracteri凶sticas lo囚gicas que poseen los tipos de datos y el nombre de los campos que se van a utilizar en el sistema, estos incluyen nombre de la tabla, campos, tipos de datos, llaves primarias y fora冈neas.

\subsubsection{Esta冈ndar de Interfaces del Sistema}

Fue definido mediante reuniones con el Product Owner, las cuales deben es- tar regidos a lineamientos de aceptacio囚n, garantizando con ello la usabilidad, navegacio®n, contenido, tiempos de respuesta, asi $₫$ como la eleccio囚n del color de la interfaz, tipo de letra y logos de la aplicacio囚n. A continuacio囚n, se muestra el bosquejo del esta冈ndar de interfaces, asi囚 como la barra de menu凶, que ayudara al manejo del sistema. Ver Figura 3.

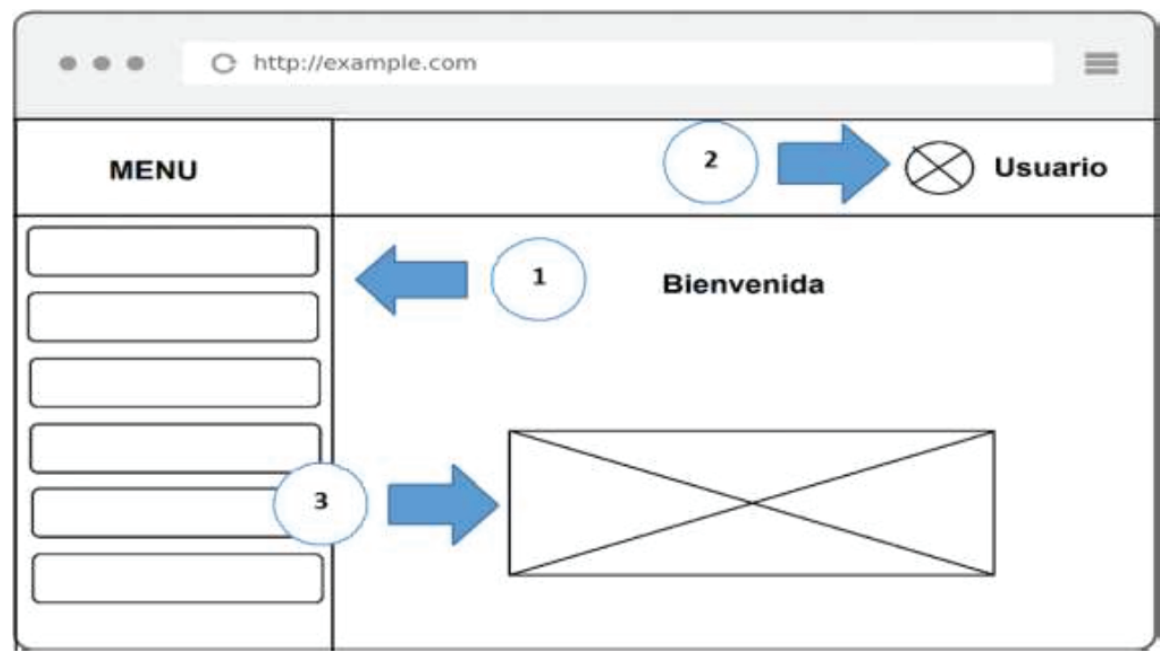

Figura 3: Bosquejo Estándar de la Interfaz. 1. Menú General del Sistema con Funciones del Sistema; 2. Credenciales del Usuario en el Sistema; and 3. Área de interacción y visualización de Mapas.

Luego de realizar el bosquejo de las interfaces que utilizara冈 el sistema, se muestra el resultado final de las interfaces disen冈adas te凶cnicamente y cumpliendo con cada una de las exigencias de usabilidad planteadas por el usuario final. Ver Figura 4. 


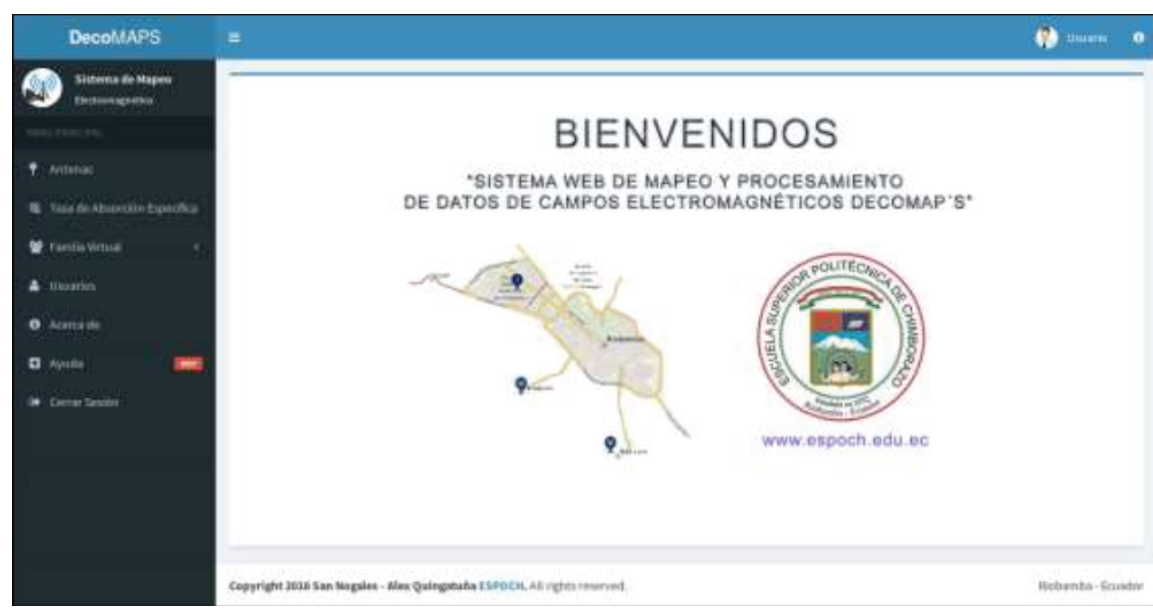

Figura 4: Diseño Estandar de la Interfaz.

\subsubsection{Desarrollo de las Historias de Usuario}

Representan los requisitos provenientes del Sprint Backlog, estas historias cuentan con un identificador, nombre, descripcio囚n y responsable; asi凶 como sus tareas de ingenieri冈a y pruebas de aceptaciođn, las mismas que comprueban el correcto cumplimiento de la historia de usuario. Para ello se describe mediante tablas el proceso de realizaciođn de una historia de usuario. La tabla II muestra un ejemplo.

TABLA 2: Historia de Usuario 01.

\begin{tabular}{|c|c|}
\hline \multicolumn{2}{|c|}{ Historia de Usuario } \\
\hline ID: HU-01 & Nombre de la Historia: Registro de Usuarios \\
\hline Usuario: Desarrollador & Sprint: 03 \\
\hline Fecha Inicio: 23/11/2015 & Fecha Fin: 24/11/2015 \\
\hline \multicolumn{2}{|c|}{$\begin{array}{l}\text { Descripción: Como desarrollador del Sistema necesito generar un proceso que } \\
\text { permita registrar usuarios }\end{array}$} \\
\hline
\end{tabular}

\subsection{SCRUM - Fase de Finalización}

En esta fase se detallan las actividades realizadas para la finalizaciođn del desarrollo del sistema DECOMAPS, para el mismo se establecioß el Sprint denominado BurnDown Chart, el cual consiste en un građfico de trabajo pendiente a lo largo del tiempo

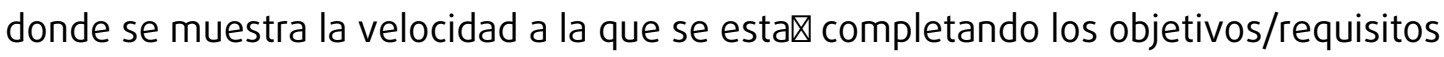
planteados al comienzo del desarrollo del sistema, adema囚s, permitio $\mathbb{Q}$ extrapolar si los desarrolladores completaron el trabajo en el tiempo estimado con satisfacciođn. 


\subsubsection{Sprint BurnDown Chart}

Al concluir con el desarrollo de los Sprint planificados se presenta mediante gra冈ficos estadi冈sticos de trabajo Sprint BurnDown Chart, el mismo que muestra la velocidad del proyecto. Ver Figura 5. El građfico esta囚 representado por dos li冈neas, que muestran los puntos ideales al planteamiento inicial del proyecto (li凶nea azul) en comparacio囚n con los puntos actuales (li冈nea naranja).

\section{Análisis de Resultados}

En esta sección analizaremos los resultados obtenidos luego de la aplicación de las diferentes tecnologías y metodologías que ayudaron a la elaboración del sistema informático DECOMAPS, para ello se realizo囚 la evaluación de la funcionalidad y usabilidad, haciendo uso del estándar ISO/IEC 9126-3 para determinar su calidad.

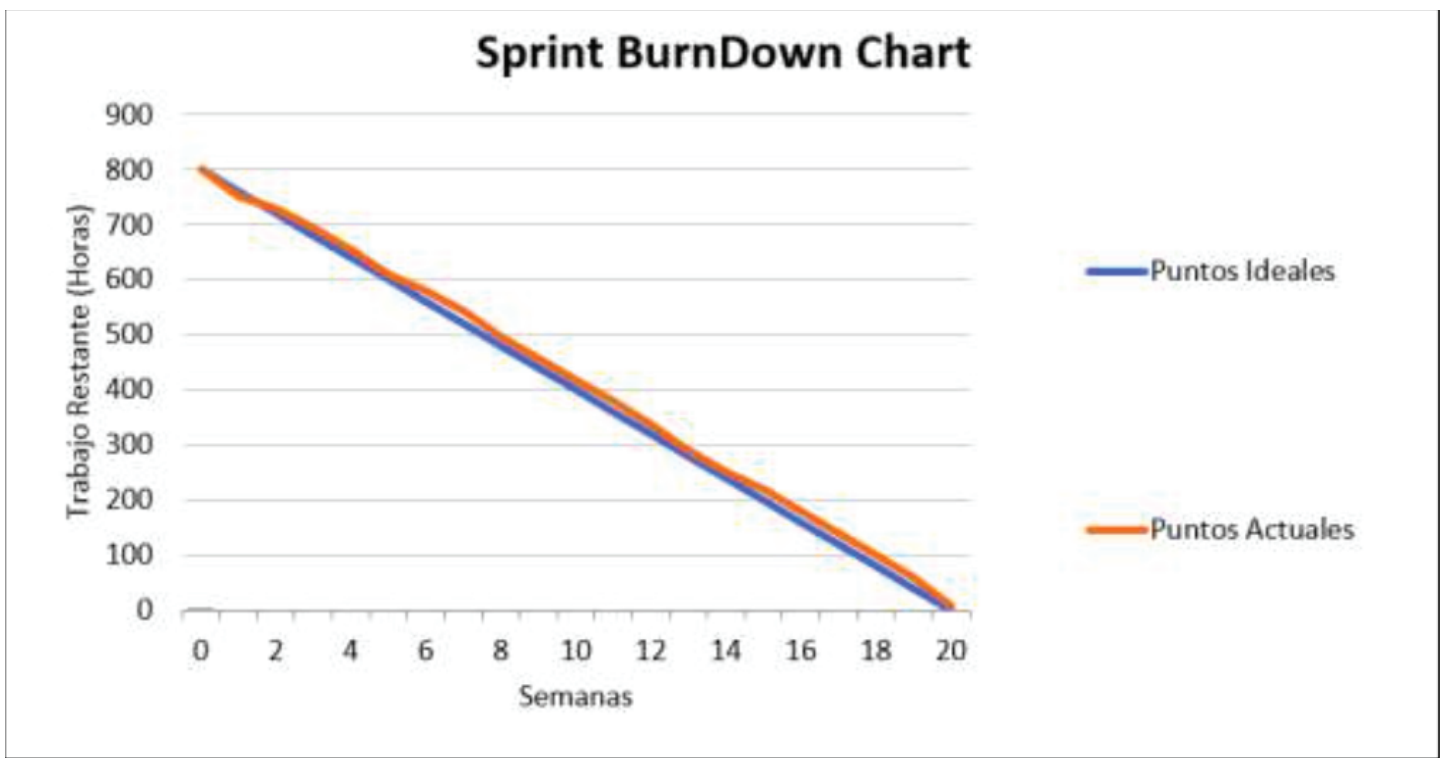

FiguRA 5: Diseño Estandar de la Interfaz.

Se decidió utilizar el ISO/IEC 9126-3 debido a varios motivos, entre ellos: el sistema que estamos desarrollando no es un software ejecutable sino más bien un Sistema Web, y lo más importante que, al utilizarse la Metodología SCRUM, era necesario ir evaluando el funcionamiento de cada uno de sus módulos durante cada una de las fases de desarrollo, así podíamos iniciar acciones correctivas temprano en el ciclo de desarrollo. 


\subsection{Requerimientos de Calidad}

Son un conjunto de sub características propias de la funcionalidad y usabilidad para la evaluación de la calidad del sistema DECOMAPS, según el estándar ISO/IEC 9126-3, se debe establecer parámetros y métricas que permitan identificar el cumplimiento de la calidad en el sistema. La tabla III, describe los parámetros y los niveles bajo los cuales se evaluó la funcionalidad y usabilidad del sistema DECOMAPS.

TABLA 3: Parámetros y Niveles de Evaluación del Sistema.

\begin{tabular}{l|l|l|c|}
\hline Característica & Subcaracterística & $\begin{array}{l}\text { Aceptación } \\
\text { Requerida }\end{array}$ & $\begin{array}{c}\text { Rango de } \\
\text { Aceptación }\end{array}$ \\
\hline Funcionalidad & Adecuidad & Alta & $0.71-1.00$ \\
\hline & Exactitud & Alta & $0.71-1.00$ \\
\hline & Interoperatividad & Media & $0.36-0.70$ \\
\hline & Seguridad & Alta & $0.71-1.00$ \\
\hline Usabilidad & Conformidades & Media & $0.36-0.70$ \\
\hline & Entendimiento & Alta & $0.71-1.00$ \\
\hline & Aprendizaje & Alta & $0.71-1.00$ \\
\hline & Operabilidad & Media & $0.36-0.70$ \\
\hline & Atracción & Baja & $0.00-0.35$ \\
\hline & Conformidad & Media & $0.36-0.70$ \\
\hline
\end{tabular}

\subsection{Ana囚lisis de la Funcionalidad del Sistema}

El ana囚lisis de la funcionalidad del sistema DECOMAPS, se ha establecido mediante las me冈tricas de sus caracteri冈sticas de calidad (adecuidad, exactitud, interoperabilidad, seguridad, conformidad de la funcionalidad), establecidas por la norma ISO 9126-3 y descritas a continuacio®n.

\subsubsection{Adecuidad}

Mide la completitud del sistema, realizado el ana囚lisis de la me囚trica de adecuidad, se obtuvo un valor nume区rico de medicio囚n (1) con un nivel de aceptacio®n "alta", dentro del rango determinado mediante la interpretacio®n de la norma para la funcionalidad del sistema. 


\subsubsection{Exactitud}

Mide la precisio囚n del sistema, realizado el ana冈lisis de la me冈trica de exactitud, se obtuvo un valor nume区rico de medicio囚n $(0,96)$ con un nivel de aceptacio®n "alta", dentro del rango determinado mediante la interpretacio®n de la norma para la funcionalidad del sistema.

\subsubsection{Interoperatividad}

Mide la interaccio囚n entre siste- mas, realizado el ana囚lisis de la me囚trica de interoperabilidad, se obtuvo un valor nume区rico de medicio®n $(0,50)$ un nivel de aceptacioखn "media", dentro del rango determinado mediante la interpretacioßn de la norma para la funcionalidad del sistema.

\subsubsection{Seguridad}

Mide la seguridad del sistema, realiza- do el ana囚lisis de la me凶trica de seguridad, se obtuvo un valor nume区rico de medicio囚n (1) con un nivel de aceptacio囚n "alta", dentro del rango determinado mediante la interpretacio®n de la norma para el funcionamiento del sistema.

\subsubsection{Conformidad}

Mide la conformidad del sistema, realizado el ana囚lisis de la me冈trica de conformidad de la funcionalidad, se obtuvo un valor nume区rico de medicio®n $(0,66)$ con un nivel de aceptacio囚n "media", dentro del rango determinado mediante la interpretacio囚n de la norma para la funcionalidad del sistema.

\subsection{Ana区lisis de la Usabilidad del Sistema}

\subsubsection{Entendimiento}

Herramientas evidentes del sistema, realizado el ana囚lisis de la me区trica de entendimiento, se obtuvo un valor nume区rico de medicio®n $(0,88)$ con un nivel de aceptacio囚n "alta", dentro del rango determinado mediante la interpretacio囚n de la norma para la usabilidad del sistema. 


\subsubsection{Aprendizaje}

Precisio®n del sistema, realizado el ana囚lisis de la me凶trica de aprendizaje, se obtuvo un valor nume囚- rico de medicio囚n $(0,70)$ con un nivel de aceptacio囚n "media", dentro del rango determinado mediante la interpretacio囚n de la norma para la usabilidad del sistema.

\subsubsection{Operabilidad}

Control del sistema, realizado el ana囚lisis de la me冈trica de operabilidad, se obtuvo un valor nume冈rico de medicio囚n $(0,60)$ con un nivel de aceptacio®n "media", dentro del rango determinado mediante la interpretacio囚n de la norma para la usabilidad del sistema.

\subsubsection{Atraccio $囚 n$}

Disen囚o del sistema, realizado el ana冈lisis de la me冈trica de atraccio囚n, se obtuvo un valor nume冈rico de medicio囚n $(0,70)$ con un nivel de aceptacio囚n "media", dentro del rango determinado mediante la interpretacioØn de la norma para la usabilidad del sistema.

\subsubsection{Conformidad}

Conformidad del sistema, realizado el análisis de la métrica de conformidad de la usabilidad, se obtuvo un valor numérico de medición $(0,66)$ con un nivel de aceptación "media", dentro del rango determinado mediante la interpretación de la norma ISO 9126-3 para la usabilidad del sistema.

\subsection{Análisis de la Calidad del Sistema}

Los datos anteriores de funcionalidad muestran los valores entre los niveles requeridos y obtenidos con sus métricas para realizar la evaluación de la funcionalidad del sistema. Podemos decir que el sistema DECOMAPS es 93,64\% funcional, calculo obtenido de la suma de los valores numéricos de las métricas, aplicada una regla de tres simple con su valor porcentual.

$$
\text { Total }=(100 * 4,12) / 4,40
$$


La Figura 6, muestra en barras los valores requeridos (rojo) en comparación con los valores obtenidos (azul) con el fin de tener una visión más amplia de la funcionalidad del sistema DECOMAPS.

Los datos anteriores de usabilidad muestran los valores entre los niveles requeridos y obtenidos con sus métricas para realizar la evaluación de la funcionalidad del sistema. Podemos decir que el sistema DECOMAPS es 94,40 \% usable, calculo obtenido de la suma de los valores numéricos de las métricas, aplicada una regla de tres simple con su valor porcentual.

$$
\text { Total }=(100 * 3,54) / 3,75
$$

La Figura 7, muestra en barras los valores requeridos (rojo) en comparación con los valores obtenidos (azul) con el fin de tener una visión más amplia de la usabilidad del sistema DECOMAPS.

El análisis de la usabilidad del sistema DECOMAPS, ha sido establecido mediante el uso de métricas de sus características de calidad (entendimiento, aprendizaje, operabilidad, atracción, conformidad de la usabilidad), establecidas por la norma ISO 9126-3 aplicada a una encuesta.

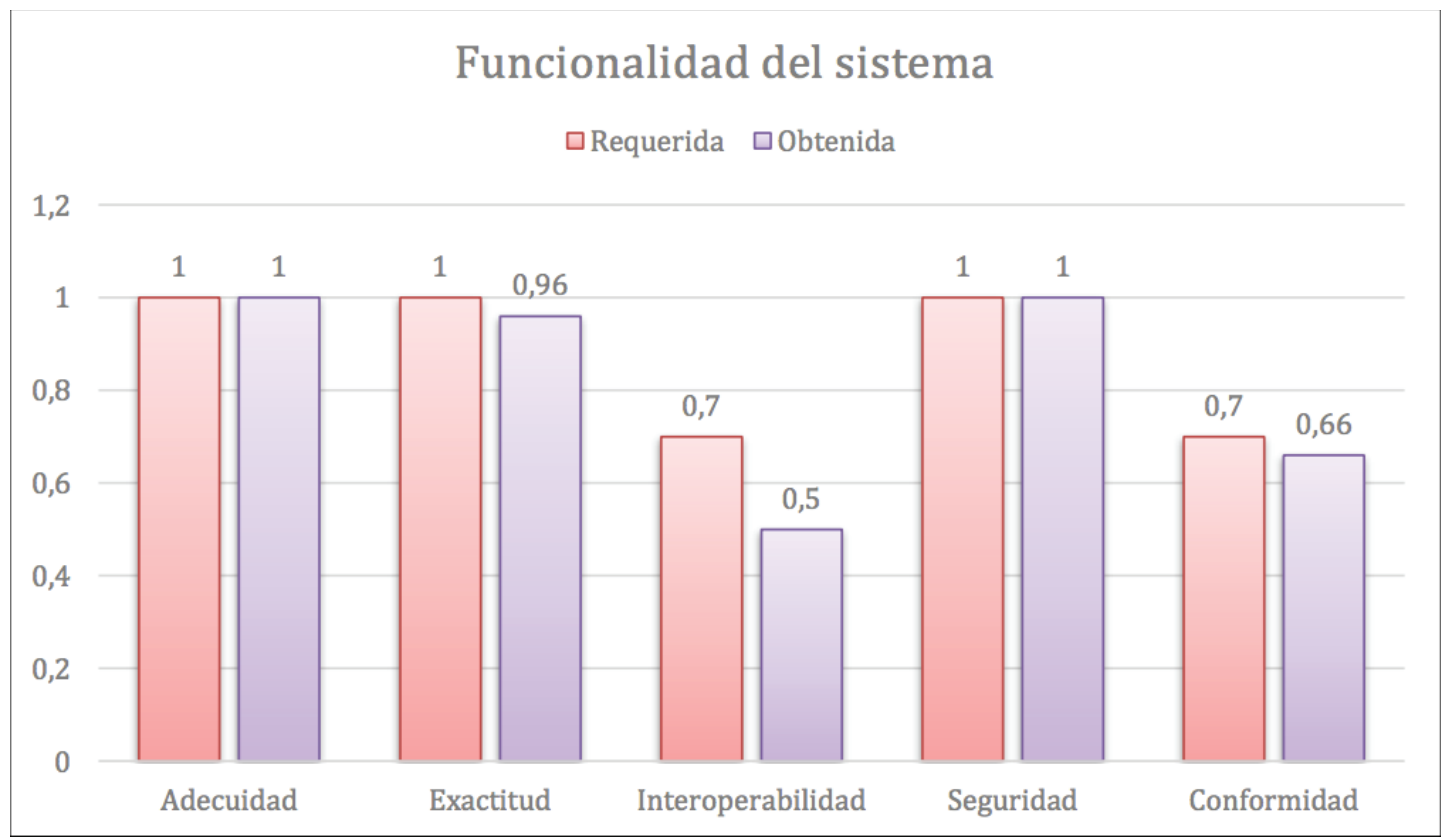

FIGURA 6: Evaluación de la funcionalidad del sistema. 


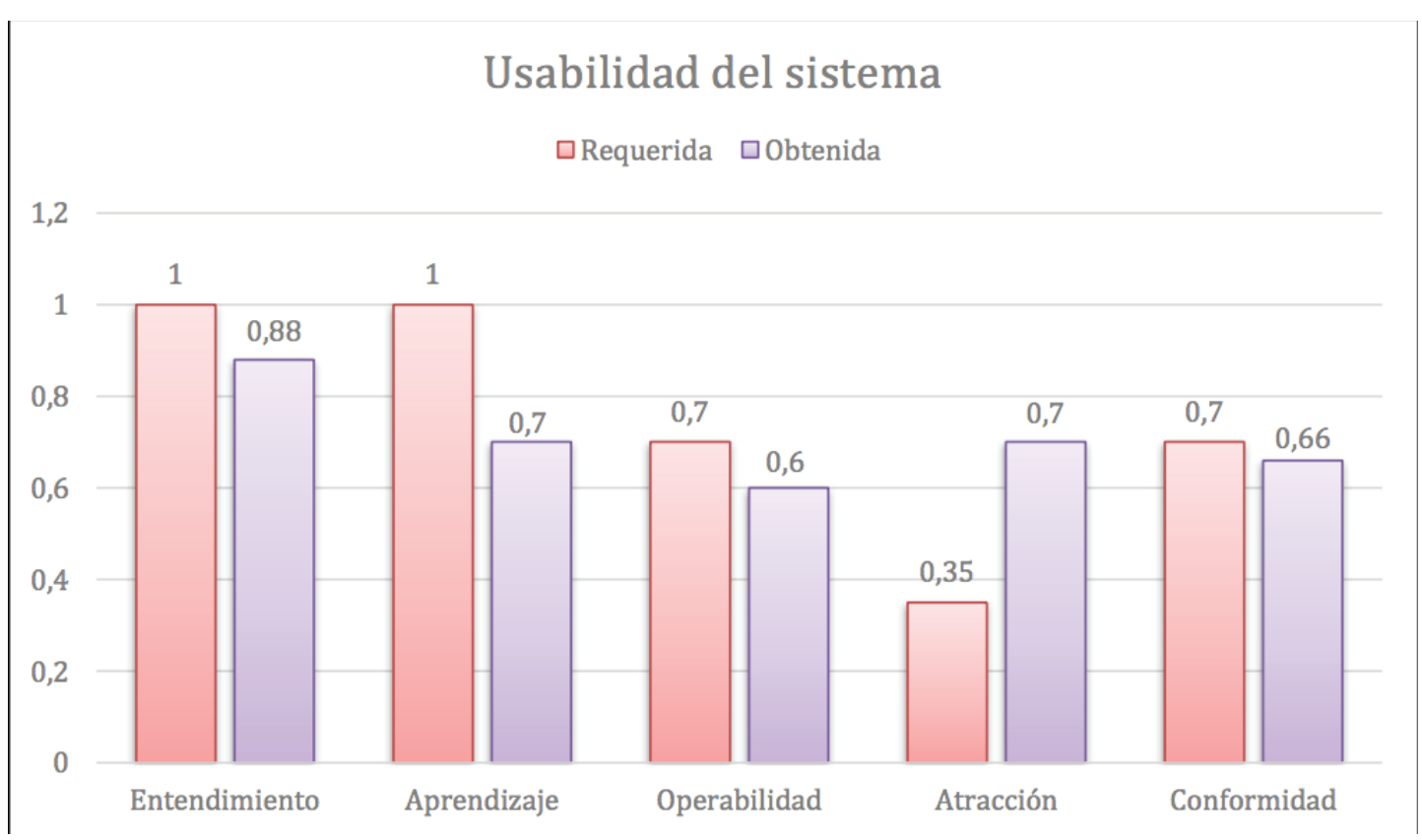

FIGURA 7: Evaluación de la funcionalidad del sistema.

\section{Conclusiones}

La versatilidad que nos brinda el desarrollo de aplicaciones web georeferencial mediante el uso de la plataforma Opengeo Suite basado en Java, ha hecho posible automatizar completamente los procesos de interpretación de datos de mapeo electromagnético que tradicionalmente se los realizaban de forma manual, con las consiguientes ventajas en confiabilidad, rapidez y precisión para la interpretación de estos datos a favor del estudio.

Una vez finalizado el desarrollo del Sistema Web Basado en Tecnologías GIS para el Mapeo de EMF de la Ciudad de Riobamba, se realizo® una evaluación de funcionalidad y usabilidad del producto final mediante las métricas de calidad, establecidas por la norma ISO 9126-3, donde se determino囚 que el sistema DECOMAPS es 93,64 \% funcional y que además cuenta con un $94,40 \%$ en usabilidad del sistema.

No es posible establecer aún cuales son las limitaciones del Sistema Web implementado debido a que aún no se ha levantado la información sobre niveles de radiación en la ciudad de Riobamba, estas mediciones se están realizando a la par del desarrollo de esta aplicación.

Este trabajo de investigación presenta un aporte técnico en el desarrollo futuro de proyectos que involucren el uso de herramientas GIS y sistemas WEB, pero sobretodo, 
este sistema servirá para determinar si los niveles de radiación electromagnética emitidos por las estaciones de telefonía causan problemas en la salud de los habitantes de esta urbe.

Como trabajos futuros, esperamos mejorar el procedimiento de programación del sistema Web basado en GIS de tal manera que sea versátil y funcional, buscando nuevas plataformas o herramientas informáticas que contribuyan a la optimización del proceso de mapeo electromagnético, además, realizar las respectivas pruebas de funcionamiento del Sistema Web con la información de medición de niveles de radiación EMF en la ciudad de Riobamba.

\section{Reconocimiento}

Los autores agradecen el patrocinio de la Escuela Superior Politécnica de Chimborazo (ESPOCH), Facultad de Informática y Electrónica, el apoyo de la Universidad de Oriente de Cuba (UO) y a quienes revisaron este trabajo gracias por sus valiosos comentarios.

\section{Referencias}

[1] J. C. Jimenez, "Antenas de telefoni冈a celular y salud", 2001.

[2] S.A. Cindy, Sage. M.A., "Summary for the public", BiolnitiativeWorking Group, Tech. Rep. 2012 Supplement, Section 1, 2012.

[3] P. Vecchia, R. Matthes, G. Ziegelberger, J. Lin, R. Saunders, and A. Swerdlow, "Exposure to high frequency electromagnetic fields, biological effects and health consequences (100 khz-300 ghz)", no. ICNIRP 16/2009, 2009.

[4] ITU-Telecommunication-Standardization-Sector, "Guidance for assessment, evaluation and monitoring of human exposure to radio frequency electromagnetic fields", International Telecommunication Union, Tech. Rep. K.91, 2012.

[5] G. Aponte, A. Escobar, C. Pinedo, and G. Arizabaleta, "Medicio囚n de campos electromagne冈ticos en la ciudad de Cali", Informacio囚n Tecnolo冈gica, vol. 18, no. 3, pp. 39-47, 2007.

[6] L. J. Heredia, "Estudio de los niveles de radiacio囚n electromagne区tica no ionizante producidas por las antenas de radio, televisioßn y estaciones base de telefoni凶a celular en varias zonas de la ciudad de Riobamba", http://dspace.espoch.edu.ec/ bitstream/123456789/3238/1/98Tooo40.pdf, Marzo 2014.

[7] F. A. Sarri冈a, "Sistemas de informacio囚n geogra冈fica", 2014. 
[8] M.G. Delgado, C.D. Castillo, F.E. Martinez, V.R. Espinoza, and M.S. Garcia, "Caracterizacio囚n de los riesgos ambientales para la salud: puesta a punto de un SIG en dos distritos del sureste de madrid", 2005.

[9] M. Marin囚o. Aplicacio®n de GIS (sistema de informacio囚n geogra冈fica) al relevamiento y ana囚lisis del patrimonio arquitecto囚nico y urbano., [Online]. Available: www.unne.edu.ar/unnevieja/Web/cyt/cyt/2001/2-Humanisticas/H-028.pdf

[10] A. Morales. (2015) Que区 es opengeo suite? [Online]. Available: http://mappinggis. com/2012/05/que-es-opengeo-suite

[11] I. Turton and F. Gasdorf. (2011) Geoserver. [Online]. Available: http://live.osgeo.org/ es/overview/geoserver_overview.html

[12] OpenLayers. (2017) [Online]. Available: https://openlayers.org/

[13] A. Marquez. "PostGIS Essentials", 2015

[14] Oracle. (2015) Que冈 es la tecnologi冈a java y para que囚 la necesito? [Online]. Available: https://www.java.com/es/download/faq/whatis_java.xml

[15] The-Apache-Software-Foundation, "Apache tomcat," 2017. [Online]. Available: http://tomcat.apache.org

[16] Calendamaia. (2015) Eclipse IDE. [Online]. Available: http://www.genbetadev.com/ herramientas/eclipse-ide

[17] M. A. A囚lvarez. (2014) Que区 es MVC? [Online]. Available: http://www. desarrolloweb.com/articulos/que-es-mvc.html

[18] C. Gutierrez. (2014) Para que囚 sirve el SCRUM en la metodologi冈a a囚gil? [Online]. Available: http://www.i2btech.com/blog- i2b/techdeployment/para-que-sirve-elSCRUM-en-la-metogologia-agil

[19] I. O. for Standardization, "ISO/IEC TR 9126-3:2003 software engineering -product quality- part 3: Internal metrics", 2003. [Online]. Available: https://www.iso.org/ standard/22891.html 